\title{
A STUDY OF MEMORY EFFECT OF PARTIAL DISCHARGES
}

\author{
YL Madhavi and MN Narayanachar
}

\begin{abstract}
From the computed conditional pulse amplitude and phase distributions it is shown that, as the gap spacing is reduced the charging of dielectric surface affects the corona pulse-time distribution which becomes significant at lower gap spacings. Most probable values are found to be the best statistical indicators for interpreting the results.
\end{abstract}

\section{Introduction}

Partial discharge (PD) phenomena which occur both in the presence and absence of solid dielectrics are inherently stochastic processes that exhibit significant statistical variability in such characteristics as pulse amplitude and phase of occurrence. The statistical behaviour of PD is governed primarily by memory effects such as associated with charge deposited by $\mathbb{D}$ on the dielectric snrfacc. When ac generated PD occur near the dielectric surface the predominant memory effect may be due to surfacc charge deposition [1-3].Much work has been done in interpreting the stochastic nature of PD phenomona. It is shown that the sum amplitude of the discharges on the positive half cyclc $Q^{+}$ affect the phase of occurrence $\phi^{-}$of the first pulse in the negative half cycle. This is shown to be true in the case of sccontl negative pulse too. The unconditional PD amplitude and phase distributions are shown to be sensitive to relatively small physical or chemical changes occuring in the gap spacing, such as might result from interaction of the discharge with the surface. The sensitivity to non-stationary behaviour is much less evident in conditional distributions. In tho present work the relative effoct of the first negative pulse $q_{1}^{-}$on the second negative pulse $q_{2}^{-}$has been studied by considering their magnitude and the time separation At distributions.

\section{Experimental arrangement and procedure}

Experiments wcre performed in a controlled environment using point-planc configuration, with and without a perspex dielectric at various voltagc levels and at different gap spacings: Straight detection method of PD detection was used: The sample was placed in a normal oven wherein standard ambient conditions were maintained following the methods given in IS : 2260 1973, Appendix A, (Clansc 3.1.1). The temperaturo was $27 \pm 2^{\circ} \mathrm{C}$ and the humidity $70 \pm 5 \%$ during the course of experiments.

The high voltage $50 \mathrm{~Hz}$ ac setup was found to be discharge free upto $10 \mathrm{kV}$. Voltages of $1.05,1.1$ and 1.2 times the inception voltage were applied. The gap spacings varied from $1 \mathrm{~mm}$ to $5 \mathrm{~mm}$ with perspex dielectric. Phase resolved pulse height analyser was used for measuring the amplitude and phase of the discharge pulses. Each record was taken for a time duration of $1 \mathrm{~min}$ and 10 such records were taken at 2 min interval. The procedure was repeated for point-planc at $5 \mathrm{~mm}$ spacing without the perspex dielectric.

\section{Experimental results and analysis}

In order to understand the basic phenomena, it is essential to study the stochastic nature of the PD behaviour at different gap spacings and at different voltage levels. As very few pulses occurred at inception voltage level, the experiments were condrictcd at overvoltages and data collccted for a sufficient time duration so that a proper statistical analysis could be made. In the case of perspex dielectric surface there wcrc a small number of pulses in the positive hall cycle followed by a large number of pulses in the negative half cyclc, whoreas in the case of point-planc, the pulses in the positive half cyclc wcre negligible. From the recorded distributions it was found that the magnitude and phase of occurrence of all the pulses occurring in the negative half cycle are distributed quantities. For the purpose of analysis only the distributions of the magnitude and phase of occurrence of the first and second pulses in the negative half cycle were considered. From the computed conditional amplitude and phase of occurrence distributions the correlations between successive pulses can be determined. The paramctcrs chosen in the present work arc $q_{1}^{-}, q_{2}^{-}$and $A t$. From the data obtained the following conditional distributions were computcd,

$\mathrm{P} 1\left(q_{2}^{-} / q_{1}^{-}\right)$: gives the probability of occurrcrice of $q_{2}^{-}$ given $q_{1}^{-}$.

$\operatorname{P1}\left(\Delta t / q_{1}^{-}\right)$: gives the probability of occurrence of $q_{2}^{-}$ in a time At given $q_{1}^{-}$. 


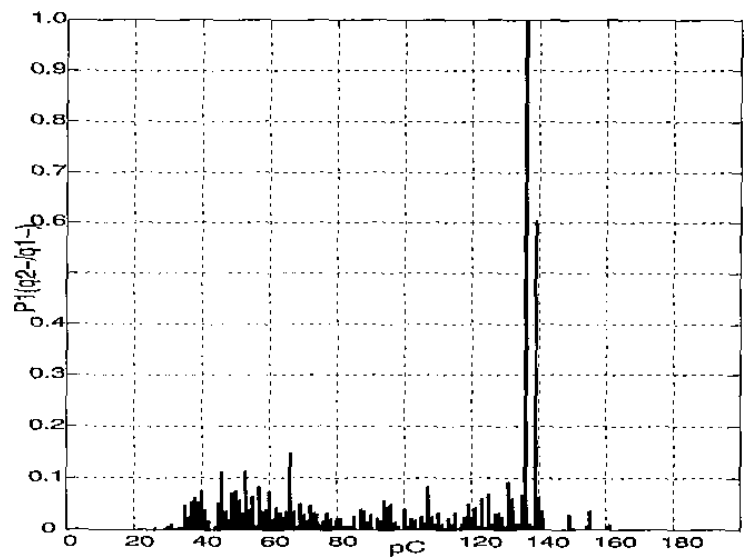

Fig. 1. $\mathrm{P} 1\left(q_{2}^{-} / q_{1}^{-}\right)$for Point-Plane $5 \mathrm{~mm} \mathrm{GAP}, 1.1 \mathrm{Vi}$

$\mathrm{P} 1\left(q_{2}^{-} / \Delta t\right)$ : probability of $q_{2}^{-}$occurring, following a given $A t$.

A number of distributions were computed for all the gaps and at all levels of vpltages. Typical distributions are presented in Figures 1-9. The probabilities are normalised with respect to the maximum occurring in the particular distribution. The distributions are best characterised and explained by considering only the most probable values. Figures 1-3 and Figures 46 show the conditional distributions $\mathrm{P} 1\left(q_{2}^{-} / q_{1}^{-}\right)$and $\mathrm{P} 1\left(\Delta t / q_{1}^{-}\right)$respectively, for the most probable value of $q_{1}^{-}$. Figures 7-9 show the conditional distributions P1 $\left(q_{2}^{-} / \Delta t\right)$ for the most probable At. We get a number of distributions $\mathrm{P} 1\left(q_{2}^{-} / q_{1}^{-}\right)$and $\mathrm{P} 1\left(\Delta t / q_{1}^{-}\right)$for different windows of $q_{1}^{-}$. Similarly we obtain a number of conditional distributions $\mathrm{P} 1\left(q_{2}^{-} / \Delta t\right)$ for different windows of At. From Figures 1-6, we can pick out the most probable $q_{2}^{-}$and the most probable At given the most probable $q_{1}^{-}$. These values are given in Table-I for all the cases. From the distributions shown in Figures 7-9 one can find the most proabable $q_{2}^{-}$for the most probable $q_{2}^{-}$. The product of $P 1_{\text {max }}\left(q_{2}^{-} / \Delta t\right)$ and $\mathrm{P}(\Delta t)$ is a joint probability which indicates the probability of the most probable $q_{2}^{-}$occurring in a particular window At where, $\mathrm{P}(\Delta t)$ is the unconditional distribution. Such joint probabilities were worked out for all the gaps and at all voltage levels. Table-I1 gives a typical case where joint probabilities are given for gap spacings of $1 \mathrm{~mm}$ and $5 \mathrm{~mm}$ with perspex dielectric and for $5 \mathrm{~mm}$ without dielectric at the same voltage level(1.1Vi). Table-I11 gives only the maximum of the joint probabilities and the respective window At. Note that At of $\mu$ s corresponds to $3.14 \times 10^{-4} \mathrm{rad}$.

\section{Discussion}

Interpretation of the results obtained from the analysis forms an important part of this discussion. From Figures 1-6 it can be seen that; following a most probable discharge $\overrightarrow{q_{1}^{-}}$, there is a most probable $q_{2}^{-}$and
TABLE I

$V_{5 p l}=3.92 \mathrm{KV}, V_{5}=3.69 \mathrm{KV}, V_{4}=4.12 \mathrm{KV}, V_{3}=3.32 \mathrm{KV}$,
$V_{2}=3.84 \mathrm{KV}, V_{1}=2.55 \mathrm{KV}$

\begin{tabular}{|c|c|c|c|}
\hline $\begin{array}{c}\text { gap } \\
\mathrm{mm}\end{array}$ & $\begin{array}{c}q_{1}^{-} \\
\mathrm{pC}\end{array}$ & $\begin{array}{c}q_{2}^{-} \\
\mathrm{pC}\end{array}$ & $\begin{array}{c}\Delta t \\
\mu \mathrm{s}\end{array}$ \\
\hline $5, \mathrm{pt}-\mathrm{plane}$ & & & \\
$1.05 V_{5 p l}$ & 65 & 70 & 80 \\
$1.1 V_{5 p t}$ & 135 & 140 & 80 \\
\hline $5, \mathrm{pt}-\mathrm{pspx}$ & & & \\
$1.05 V_{5}$ & 115 & 110 & 160 \\
$1.1 V_{5}$ & 165 & 175 & 80 \\
$1.2 V_{5}$ & 165 & 170 & 80 \\
\hline $4, \mathrm{pt}-\mathrm{pspx}$ & & & \\
$1.05 V_{4}$ & 95 & 90 & 160 \\
$1.1 V_{4}$ & 115 & 120 & 120 \\
$1.2 V_{4}$ & 115 & 120 & 80 \\
\hline $3, \mathrm{pt}-\mathrm{pspx}$ & & & \\
$1.05 V_{3}$ & 115 & 120 & 160 \\
$1.1 V_{3}$ & 115 & 120 & 120 \\
$1.2 V_{3}$ & 105 & 110 & 120 \\
\hline $2, \mathrm{pt-pspx}$ & & & \\
$1.05 V_{2}$ & 125 & 130 & 120 \\
$1.1 V_{2}$ & 125 & 130 & 120 \\
$1.2 V_{2}$ & 125 & 130 & 120 \\
\hline $1, \mathrm{pt}-\mathrm{pspx}$ & & & \\
$1.05 V_{1}$ & 135 & 130 & 440 \\
$1.1 V_{1}$ & 115 & 130 & 320 \\
$1.2 V_{1}$ & 125 & 130 & 440 \\
\hline
\end{tabular}

TABLE II

\begin{tabular}{|c|c|c|c|}
\hline gap, $\mathrm{mm} \rightarrow$ & $\begin{array}{c}1 \\
\text { pcrspex }\end{array}$ & 5 & $\begin{array}{c}5 \\
\text { pt-plane }\end{array}$ \\
\hline joint & 0.33 & 2.1 & 2.5 \\
probability & 1.4 & 1.8 & 1.3 \\
× $10^{-2}$ & 3.1 & 0.43 & 0.72 \\
$\downarrow$ & 3.6 & 0.25 & 0.4 \\
& 2.6 & 0.17 & 0.55 \\
& 1.3 & 0.24 & 0.4 \\
& 0.37 & 0.18 & \\
& 0.13 & 0.33 & \\
& 0.01 & 0.23 & \\
& 0.073 & 0.28 & 0.15 \\
& 0.056 & 0.16 & 0.076 \\
\hline
\end{tabular}




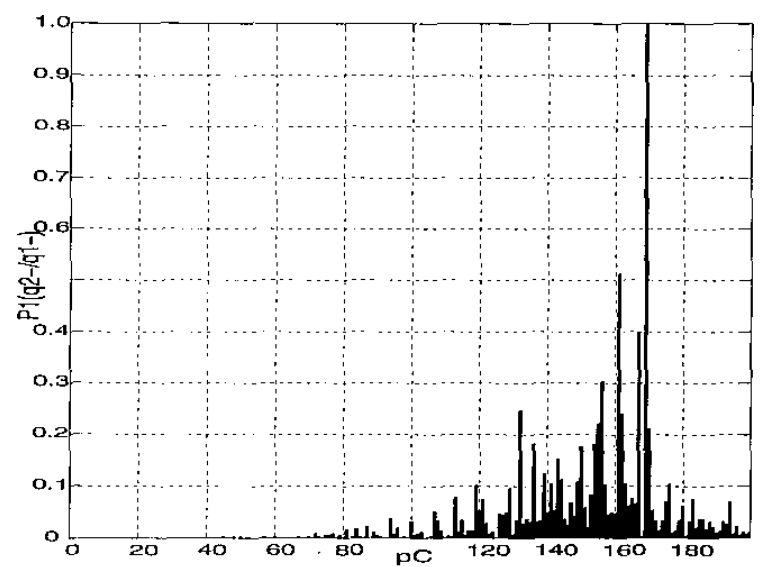

Fig. 2. $\mathrm{P} 1\left(q_{2}^{-} / q_{1}^{-}\right)$for Point-Perspex $5 \mathrm{~mm}$ GAP, $1.1 \mathrm{Vi}$

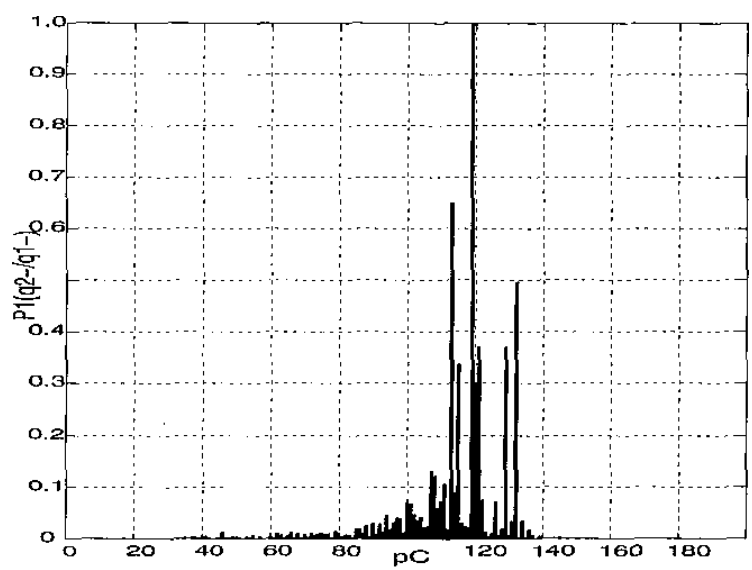

Fig. 3. P1 $\left(q_{2}^{-} / q_{1}^{-}\right)$for Point-Perspex 1mm GAP, 1.1Vi

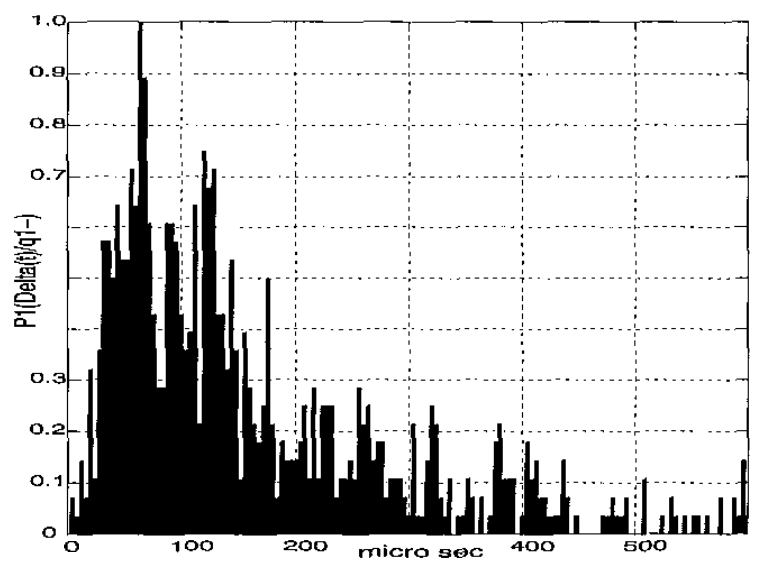

Pig. 4. $\mathrm{P} 1\left(\Delta t / q_{1}^{-}\right)$for Point-Plane $5 \mathrm{~mm}$ GAP, $1.1 \mathrm{Vi}$

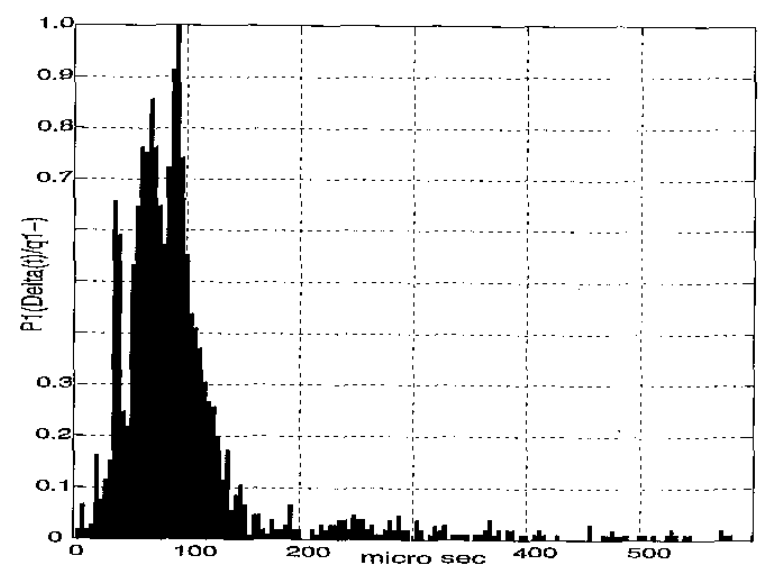

Fig. 5. $\mathrm{P} 1\left(\Delta t / q_{1}^{-}\right)$for Point-Perspex $5 \mathrm{~mm} \mathrm{GAP}, 1,1 \mathrm{Vi}$

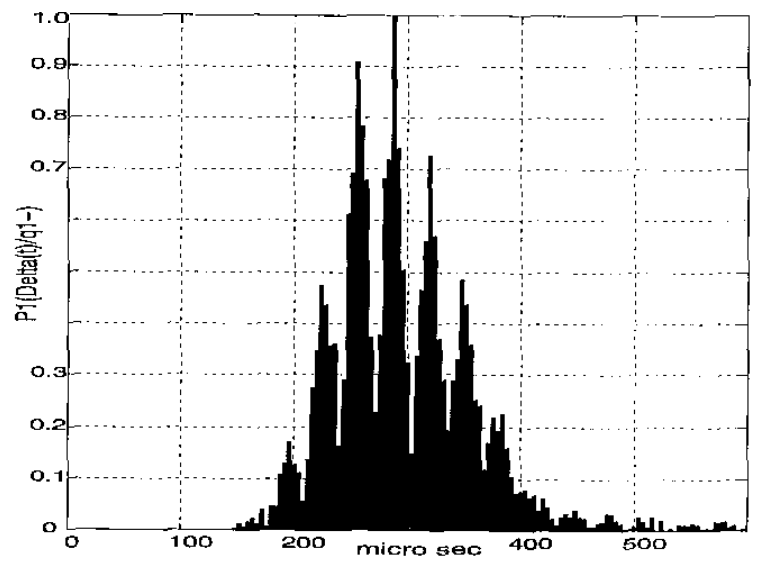

Fig. 6. $P \mathbf{1}\left(\Delta t / q_{1}^{-}\right)$for Point-Perspcx $1 \mathrm{~mm}$ GAP, $1.1 \mathrm{Vi}$

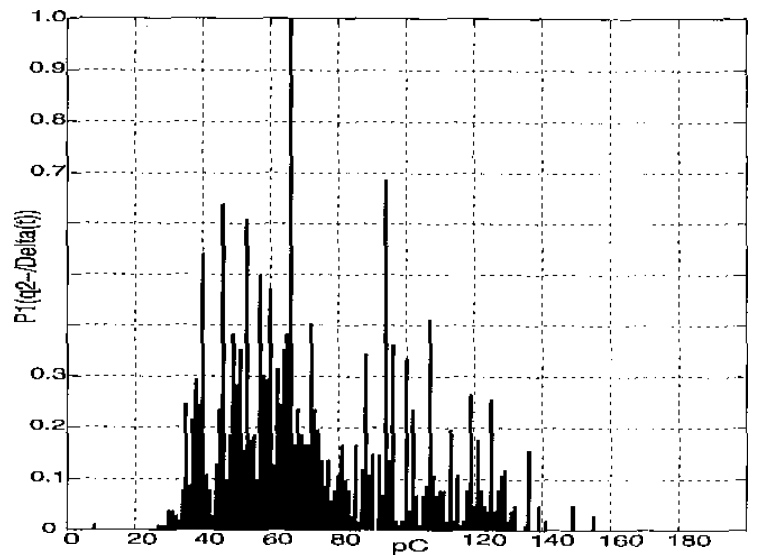

Fig. 7. $\mathrm{P} 1\left(q_{2}^{-} / \Delta t\right)$ for Point-Plane 5mm GAP, $1.1 \mathrm{Vi}$ 
TABLE III
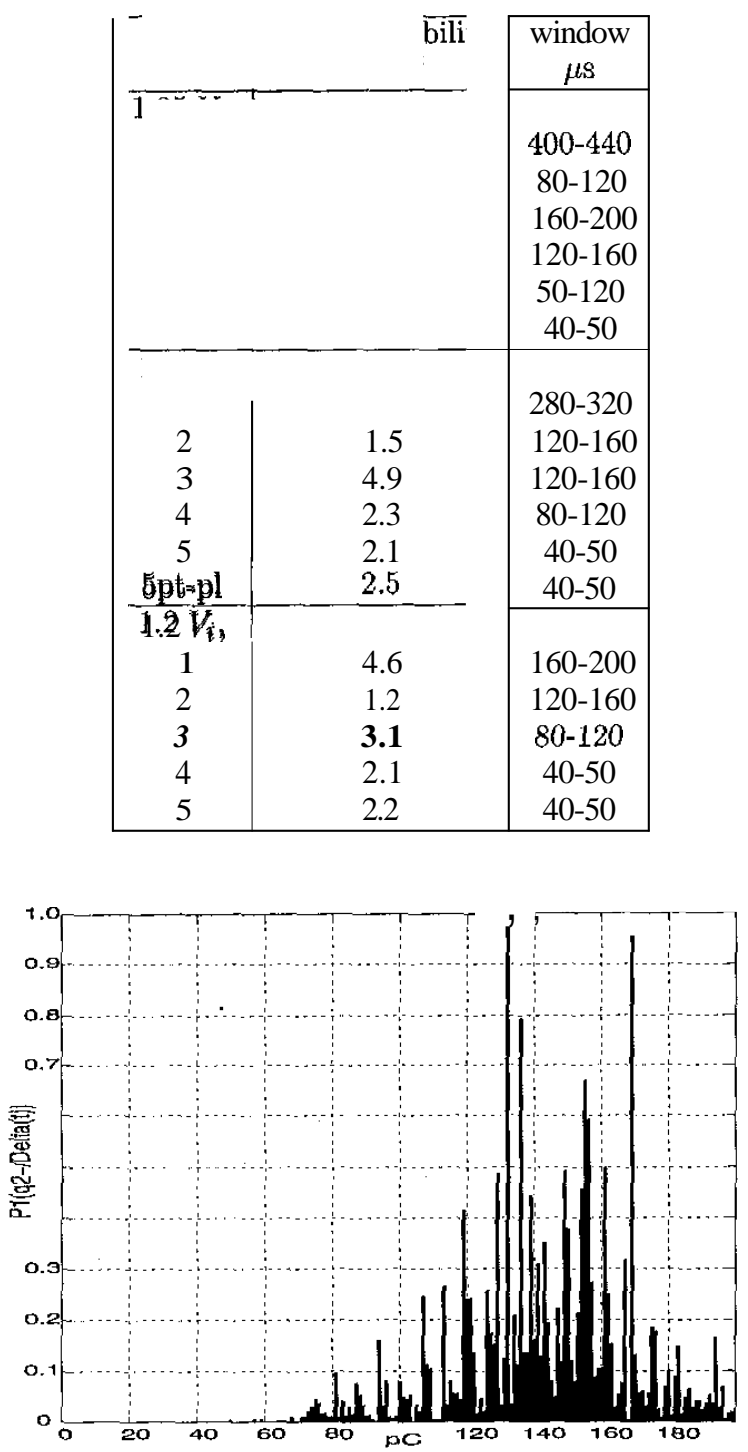

Fig. 8. $\quad \mathrm{P} 1\left(q_{2}^{-} / \Delta t\right)$ for Point-Perspex $\$ \mathrm{~mm}$ GAP, $1,1 \mathrm{Vi}$

a most probable At. However the most probable $q_{z}^{-}$ need not always follow the most probable At. But if one works out a joint probability, one can find out the most probable $q_{2}^{-}$and $A t$, following $q_{1}^{-}$. This is when the joint probability $\mathrm{P} 1\left(q_{2}^{-} / A t\right) \times \mathrm{P}(\Delta t)$ is a maximum. This value is $3.6 \times 10^{-} 2$ for $1 \mathrm{~mm}$ gap (Table-11). It can be seen from Table-I1 that,

1. the time lag increases with decrease in the gap spacing at all voltage levels. This reflects the increasing effect of spacing on the gap characteristic $s$ that might occur duc to the interaction of the discharges on the surface of the dielectric.

2. the ovcrstrcssing of the gap tends to decrease the time lag in the range 1 to $3 \mathrm{~mm}$ of the gap spacing.

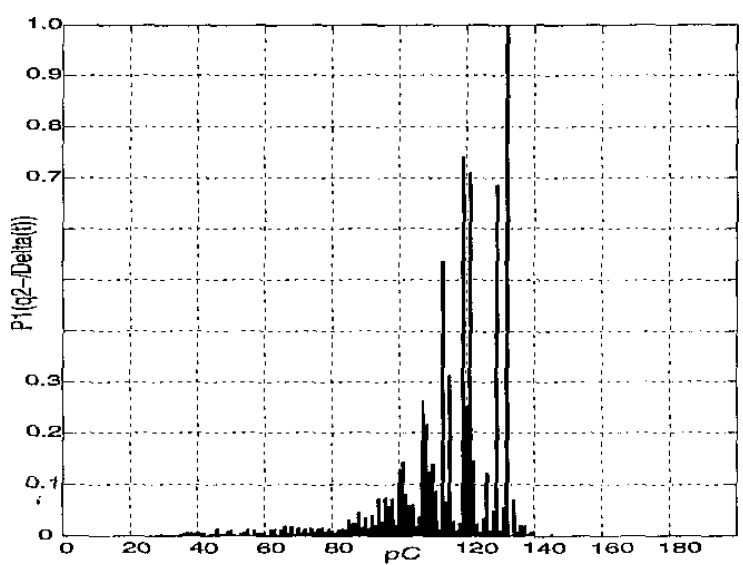

Fig. 9. $P 1\left(g_{2}^{-} / \Delta t\right)$ for Point-Perspex $1 \mathrm{~mm}$ GAP, $1,1 \mathrm{Vi}$

At higher spacing (4 and $5 \mathrm{~mm}$ ) the effect is not pronounced which fairly reflects the ineffectiveness of the dielectric surface at higher spacings.

From Table-I we can infer that,

1. the most probablc $q_{1}^{-}$increases with decrease in gap spacing.

2. there is positive correlation between $q_{2}^{-}$and $q_{1}^{-}$.

3. the value of $A t$ increases with decrease in gap spacing.

4. the time lags seem to stabilize to thier characteristic values for lower gap spacings even at overvoltages.

\section{Conclusions}

It is shown that the effect of dielectric on the recurrence of corona pulses can be studied by considering the conditional distributions of the first two pulses in the negative half cycle and the time difference between them. The analyses of the parameters show the effect of the previous discharges on the following events.

\section{References}

1. R.J.Van Brunt, E.W.Cernyar, P.Von Glahn, "Importance of Unravelling Memory Propagation Effects in Interpreting Data on Partial Discharge Statistics" , IEEE transactions on Elec.Insul., Vol.28, pp.905-916, 1993.

2. R.J.Van Brunt, M.Misakian, S.V.Kulkarni, V.K.Lakadawala, "Influence of a Dielectric Barrier on the Stochastic Behavior of Trichel-pulse Corona", IEEE transactions on Elec.Insul., Vol.26, pp.405-415, 1991.

3. P.Von Glahn and R.J.Van Brunt, "Continuous Recording and Stochastic Analysis of PD", IEEE transactions on Dielectrics and Elec.Insul., Vol.2, No.4, pp.590-601, 1995. 